\title{
Carnosol induces the osteogenic differentiation of bone marrow- derived mesenchymal stem cells via activating BMP-signaling pathway
}

\author{
Basem M. Abdallah* \\ Department of Biological Sciences, College of Science, King Faisal University, Al-Ahsa 31982, Saudi Arabia
}

\section{ARTICLE INFO}

Received October 8, 2020

Revised January 13, 2021

Accepted January 25, 2021

*Correspondence

Basem M. Abdallah

E-mail: babdallallah@kfu.edu.sa

Key Words

Adipocyte

Bone morphogenetic protein signaling

Carnosol

Mesenchymal stem cells

Osteoblast

\begin{abstract}
Carnosol is a phenolic diterpene phytochemical found in rosemary and sage with reported anti-microbial, anti-oxidant, anti-inflammatory, and anti-carcinogenic activities. This study aimed to investigate the effect of carnosol on the lineage commitment of mouse bone marrow-derived mesenchymal stem cells (mBMSCs) into osteoblasts and adipocytes. Interestingly, carnosol stimulated the early commitment of mBMSCs into osteoblasts in dose-dependent manner as demonstrated by increased levels of alkaline phosphatase activity and Alizarin red staining for matrix mineralization. On the other hand, carnosol significantly suppressed adipogenesis of mBMSCs and downregulated both early and late markers of adipogenesis. Carnosol showed to induce osteogenesis in a mechanism mediated by activating BMP signaling pathway and subsequently upregulating the expression of BMPs downstream osteogenic target genes. In this context, treatment of mBMSCs with LDN-193189, BMPR1 selective inhibitor showed to abolish the stimulatory effect of carnosol on BMP2-induced osteogenesis. In conclusion, our data identified carnosol as a novel osteoanabolic phytochemical that can promote the differentiation of mBMSCs into osteoblasts versus adipocytes by activating BMP-signaling.
\end{abstract}

\section{INTRODUCTION}

Bone mass integrity is maintained by the balance between osteoblastic bone formation and osteoclastic bone resorption $[1,2]$. Bone forming osteoblasts are recruited from bone marrowderived mesenchymal stem cells (BMSCs) via stages of cellular commitment, differentiation, osteoblast maturation and matrix mineralization [3,4]. Osteoclast, the multinucleated giant cells that resorb bone are derived from monocyte-macrophage lineage via a process, including cell proliferation, differentiation and fusion [5].

BMSCs are a group of non-hematopoietic stem cells that present in bone marrow niche and have the potential to differentiate into osteoblast, adipocyte, chondrocyte and other mesoderm- derived cell lineages [3,5-7]. Thus, identifying molecules that can direct the differentiation fate of BMSCs into osteogenic lineage will increase bone formation $[8,9]$.

The Mediterranean diet is rich with a wide variety of phytochemicals that are known to have many pharmacological properties for improving chronic diseases including cardiovascular diseases, diabetes, inflammation, and cancer [10,11]. Carnosol $\left(\mathrm{C}_{20} \mathrm{H}_{26} \mathrm{O}_{4}\right)$ is a phenolic diterpene phytochemical extracted mainly from Rosmarinus officinalis (rosemary), which representing the main constituents of antioxidant compounds of rosemary leaves $[12,13]$. Carnosol was reported for its wide range of bioactivities in vitro and in vivo, including antibacterial, anti-inflammatory, anticarcinogenic, neuroprotective and antioxidative effects [13-16]. Recently, carnosol was found to exert an antiaging

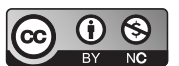

This is an Open Access article distributed under the terms of the Creative Commons Attribution Non-Commercial License, which permits unrestricted non-commercial use, distribution, and reproduction in any medium, provided the original work is properly cited. Copyright @ Korean J Physiol Pharmacol, pISSN 1226-4512, elSSN 2093-3827
Author contributions: B.M.A. conceived and designed the project, data analysis, drafting and revising the article. 
effect in $C$. elegans by significantly inhibiting the reactive oxygen species accumulation under normal or oxidative stress condition [17].

In terms of bone biology, Carnosol was reported to inhibit the catabolic mediator's gene expression of cartilage breakdown in chondrocytes via its anti-inflammatory effect to inhibit IL-6 production by osteoblast [18]. In addition, carnosol significantly inhibited the expression of pro-inflammatory cytokines and the nuclear translocation of the receptor activator of nuclear factor$\kappa \mathrm{B}(\mathrm{NF}-\kappa \mathrm{B})$ in human primary chondrocytes stimulated by IL- $1 \beta$ $[19,20]$, and to suppress the RANKL-induced osteoclast formation [21]. However, the potential role of carnosol in osteoblast differentiation of BMSCs was unknown.

In this study, we investigated the effect of carnosol on the differentiation of BMSCs into osteoblast lineage and adipocyte lineage. Our results demonstrated the dual effect of carnosol on stimulating osteogenesis and inhibiting adipogenesis via BMPdependent mechanism.

\section{METHODS}

\section{Cell cultures and reagents}

Experiments were approved by the Standing Research Ethics Committee, King Faisal University, Saudi Arabia with approval number KFU-REC/2020-01-12 .

Primary mouse BMSCs were isolated and cultured from 8-weeks-old male C57BL/6J mice as previously reported by our group [22]. In brief, bone marrow cells were flushed out from mouse tibia and femur, and then filtrated through a $70-\mu \mathrm{m}$ nylon mesh filter. Cells were cultured in RPMI-1640 medium supplemented with 12\% FBS (Gibco Invitrogen, Grand Island, NY, USA), $12 \mu \mathrm{M}$ L-glutamine (Gibco Invitrogen) and 1\% penicillin/ streptomycin (P/S) (Gibco Invitrogen). After $24 \mathrm{~h}$, non-adherent cells were removed and cultured in $60 \mathrm{~cm}^{2}$. Medium was changed every 3-4 days and cells were washed and regularly sub-cultured.

Carnosol and LDN-193189 were purchased from (SigmaAldrich Chemie Gmbh, Munich, Germany).

\section{Cell toxicity assay}

Cell toxicity was determined by measuring cell viability using MTT cell proliferation assay kit after 3 days of treatment with carnosol (Sigma-Aldrich) according to the manufacturer's instructions kit. Cells were incubated with MTT solution to metabolize to formazan and absorbance was measured at a wavelength of $550 \mathrm{~nm}$.

\section{Osteoblast differentiation}

mBMSCs were induced to differentiate into osteoblasts with osteogenic induction medium (OIM) consists of in $\alpha$-minimum essential medium ( $\alpha$-MEM; Gibco) supplemented with $10 \%$ FBS, $100 \mathrm{U} / \mathrm{ml}$ of penicillin, $100 \mathrm{mg} / \mathrm{ml}$ of streptomycin, $10 \mathrm{mM}$ $\beta$-glycerol-phosphate (Sigma-Aldrich ApS, Hamburg, Germany), and $50 \mathrm{mg} / \mathrm{ml}$ of vitamin C (Sigma-Aldrich ApS). Medium changed every third day during the differentiation course.

\section{Adipocyte differentiation}

Mouse bone marrow-derived mesenchymal stem cells (mBMSCs) were induced to differentiate into adipocytes with adipogenic-induction medium (AIM) consists of low glucose DMEM supplemented with 9\% horse serum, $450 \mu \mathrm{M}$ 1-methyl-3-isobutylxanthine (IBMX), $250 \mathrm{nM}$ dexamethasone, $5 \mu \mathrm{g} / \mathrm{ml}$ insulin (Sigma-Aldrich) and $1 \mu \mathrm{M}$ rosiglitazone (BRL 49653; Cayman Chemical, Ann Arbor, MI, USA). Medium changed every third day during the differentiation course.

\section{Quantitative of alkaline phosphatase (ALP) activity}

Cells were cultured in 96 well plate and induced with OIM as mentioned above. ALP activity was determined by incubating the cells with $1 \mathrm{mg} / \mathrm{ml}$ of P-nitro phenyl phosphate in $50 \mathrm{mM}$ $\mathrm{NAHCO}_{3}$ and $1 \mathrm{mM} \mathrm{MgCl}_{2}$ buffer (pH 9.6) at $37^{\circ} \mathrm{C}$ for $20 \mathrm{~min}$. The reaction was stopped by the addition of $3 \mathrm{M} \mathrm{NaOH}$. The absorbance of reaction was measured at $405 \mathrm{~nm}$. Cell viability was determined using the CellTiter-Blue cell viability assay. ALP activity was represented after normalization to the value of cell viability and each sample was measured in 6 replicates.

\section{ALP staining}

Cells were induced to osteogenic lineage and fixed with acetone/citrate buffer $\mathrm{pH} 4.2$ (1.5:1) for $5 \mathrm{~min}$ at room temperature. Cells were stained with Napthol-AS-TR-phosphate solution (Sigma-Aldrich ApS) for $1 \mathrm{~h}$ at room temperature. Napthol-AS-TRphosphate solution consists of Napthol-AS-TR-phosphate diluted 1:5 in $\mathrm{H}_{2} \mathrm{O}$ and Fast Red TR (Sigma-Aldrich ApS) diluted 1:1.2 in $0.1 \mathrm{M}$ Tris buffer, $\mathrm{pH}$ 9.0, after which both solutions were mixed 1:1 and applied to the cells.

\section{Alizarin Red S staining and quantification}

Cells were induced to differentiate into osteoblasts for 10-12 days and fixed with $70 \%$ ice-cold ethanol for $1 \mathrm{~h}$ at $-20^{\circ} \mathrm{C}$. Cells were stained with Alizarin Red ( $40 \mathrm{mM}, \mathrm{pH}=4$; Sigma-Aldrich ApS) for $10 \mathrm{~min}$ at room temperature. For quantification of calcium deposition, Alizarin Red S was eluted with 10\% cetylpyridinium chloride (Sigma-Aldrich ApS) for $1 \mathrm{~h}$ at room temperature. The absorbance of the eluted dye was measured at $570 \mathrm{~nm}$. 


\section{Oil Red $\mathrm{O}$ staining and quantification}

Cells were induced to differentiate into adipocyte using AIM, then fixed in $4 \%$ paraformaldehyde for $10 \mathrm{~min}$ at room temperature, and fate droplets were stained with Oil Red O $(0.5 \mathrm{~g}$ in $60 \%$ isopropanol) (Sigma-Aldrich) for $1 \mathrm{~h}$. For lipids quantification, Oil Red O was eluted from the cells with isopropanol for $10 \mathrm{~min}$ at room temperature and absorbance of the extracted dye was detected at OD $490 \mathrm{~nm}$. Oil Red O values were normalized to cell number (measured by number of viable cells).

\section{Western blot analysis}

Cells were treated without or with carnosol and collected at specific time points and lysed in cell lysis buffer (10 mM Tris$\mathrm{HCl}, \mathrm{pH}$ 7.4, 150 mM sodium chloride, 1\% NP-40, 0.1\% SDS, 1 mM EDTA, $1 \mathrm{mM}$ phenyl-methylsulfonyl fluoride, $1 \mathrm{mM} \mathrm{NaF}$, $1 \mathrm{mM} \mathrm{Na}_{3} \mathrm{VO}_{4}$ ), supplemented with protease inhibitor cocktail (Roche Diagnostics, Mannheim, Germany). $30 \mu \mathrm{g}$ of protein was separated on $8 \%-12 \%$ NuPAGE Novex Bis-Tris gel systems (Thermo Fisher Scientific, Roskilde, Germany) followed by transfer to PVDF membrane (Millipore, Bedford, MA, USA). The membrane was blocked and probed with antibodies (dil 1:1,000) and incubated with secondary anti-rabbit horseradish peroxidaseconjugated antibody (Santa Cruz Biotechnology, Aarhus, Denmark). Antibodies for Smad1/5/8 (total or phosphor) and $\beta$-Actin purchased from Santa Cruz Biotechnology, Inc. (Heidelberg, Germany). Quantification of Western blots was performed with ImageJ program.

\section{RNA extraction and real-time PCR analysis}

Total RNA was extracted from cells using TRIzol single-step method (Thermo Fisher Scientific). cDNA was synthesized from $1 \mu \mathrm{g}$ of total RNA using revertAid $\mathrm{H}$ minus first strand cDNA synthesis kit (Fermentas, St Leon-Rot, Germany) according to the manufacturer's instructions. Quantitative real time PCR was performed using Applied Biosystems 7500 Real-Time system with Fast SYBR Green Master Mix (Applied Biosystems, Foster City, CA, USA). Primer sequences for target genes were presented in Supplementary Table 1 . The expression of each target gene was normalization to $\beta$-Actin and Hprt mRNA expression as
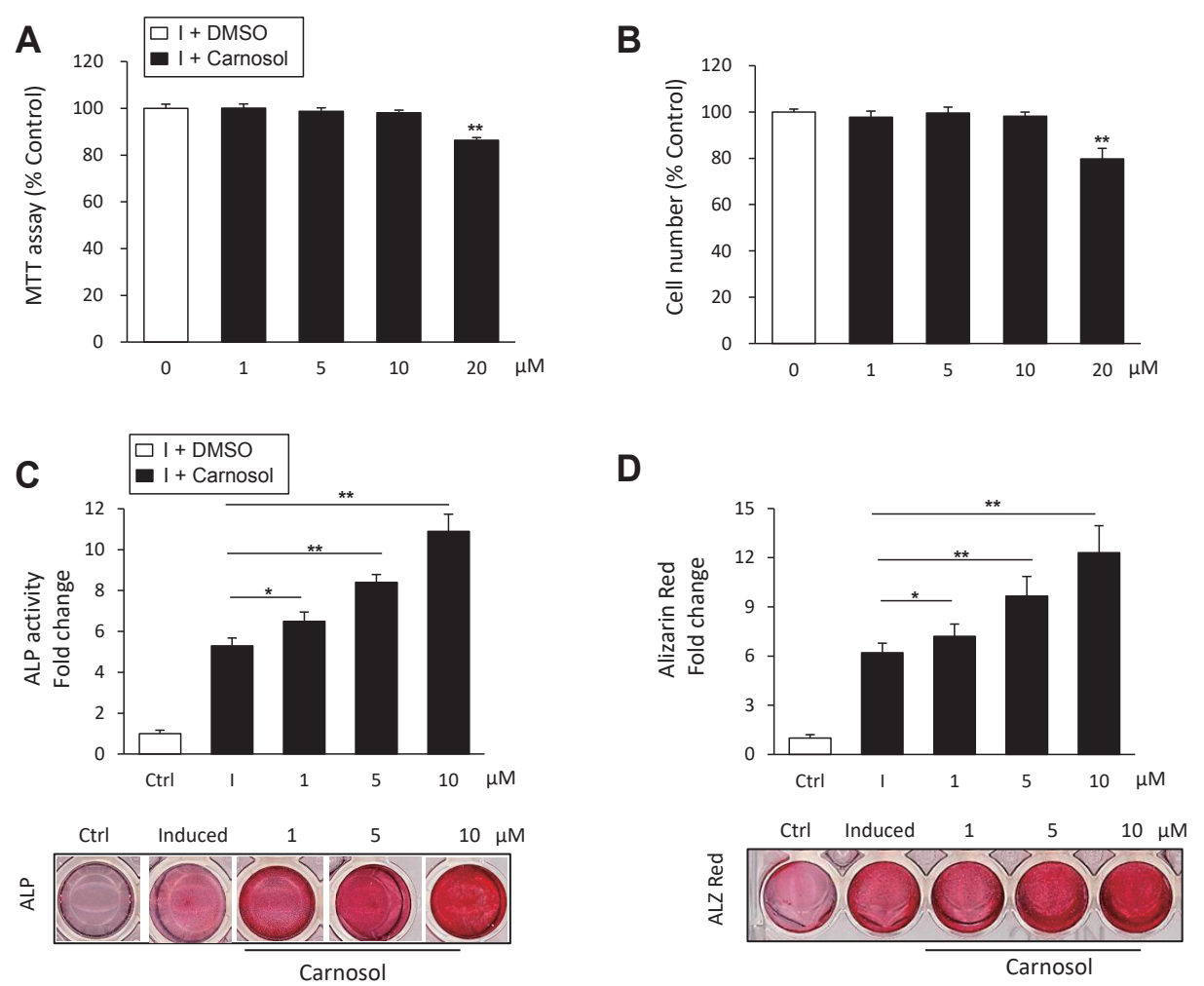

Fig. 1. Carnosol induces the differentiation of mouse bone marrow-derived mesenchymal stem cells (mBMSCs) into osteoblasts. (A) Studying the cytotoxicity of carnosol on primary culture of mBMSCs using MTT cell viability assay. (B) Studying the effect of different concentrations of carnosol on mBMSCs cell proliferation as measured by counting cell number. Cells were either non-treated (0) or treated with different doses of carnosol for 3 days. (C) Stimulatory effect of carnosol on osteoblast differentiation of mBMSCs as assessed by ALP activity quantification and (D) Alizarin red staining quantification for matrix mineralization after 6 and 12 days of induction respectively. Stained images were shown under each graph. mBMSCs were either non-induced (control, ctrl), or induced with OIM in the absence (I) or the presence of different concentrations of carnosol. Values were shown as fold change over control. Values are mean \pm standard deviation of three independent experiments $\left({ }^{*} p<0.05,{ }^{* *} p<0.005\right.$ compared to Control [0] for [A and $\mathrm{B}]$ and compared to differentiated cells without carnosol [l] for panals [C and D]). ALP, alkaline phosphatase; OIM, Osteogenic-induction medium. 
reference genes, using a comparative CT method [(1/(2delta-CT) formula, where delta-CT is the difference between CT-target and CT-reference] with Microsoft Excel 2007 [23].

\section{Statistical analysis}

All values are expressed as mean \pm standard deviation (SD), of at least 3 independent experiments. The data analyzed statistically for 2-samples using unpaired Student's t-test (2-tailed) assuming equal variation in the two groups. Differences were considered statistically significant at ${ }^{*} \mathrm{p}<0.05$, and ${ }^{* *} \mathrm{p}<0.005$.

\section{RESULTS}

\section{Carnosol promotes osteoblast differentiation of mBMSCs}

We first studied the cytotoxicity of carnosol on primary culture of mBMSCs using cell viability MTT assay and cell counting of viable cells. Carnosol showed no effect on cell viability up to concentration of $10 \mu \mathrm{M}$ as assessed by MMT assay and cell count after 3 days in culture (Fig. 1A, B). On the other hand, carnosol displayed cytotoxicity at concentration of $20 \mu \mathrm{M}$. Therefore, we used a range of carnosol concentration from 1 to $10 \mu \mathrm{M}$ for all of our subsequent experiments.

We examined the effect of carnosol on the osteoblast differentiation of mBMSCs. As shown in Fig. 1C, carnosol displayed dose-dependent stimulatory effect on ALP activity by mBMSCs during their differentiation into osteoblast lineage as compared to induced cells without carnosol (Fig. 1C). In addition, carnosol promoted matrix mineralization in mBMSCs during osteogenesis in dose-dependent manner as assessed by the quantification of Alizarin Red staining (Fig. 1D). We further studied the effect of carnosol on osteogenesis during the time course of 12 days of differentiation. Treatment of mBMSCs with carnosol $(10 \mu \mathrm{M})$ showed to stimulate the ALP activity of mBMSCs during the whole differentiation course (Supplementary Fig. 1A).

\section{Carnosol upregulates the expression of osteoblastic- related genes in $\mathrm{mBMSCs}$}

We studied further the effect of carnosol on osteogenesis at molecular level, by measuring its effect on osteogenic gene expression using qPCR. Treatment of mBMSCs during osteogenesis with carnosol showed to significantly upregulate the expression of both early (Runx2, Osx, Colla1, and Alp) and late (Ocn and Opn) osteogenic markers (Fig. 2A). In addition, carnosol significantly

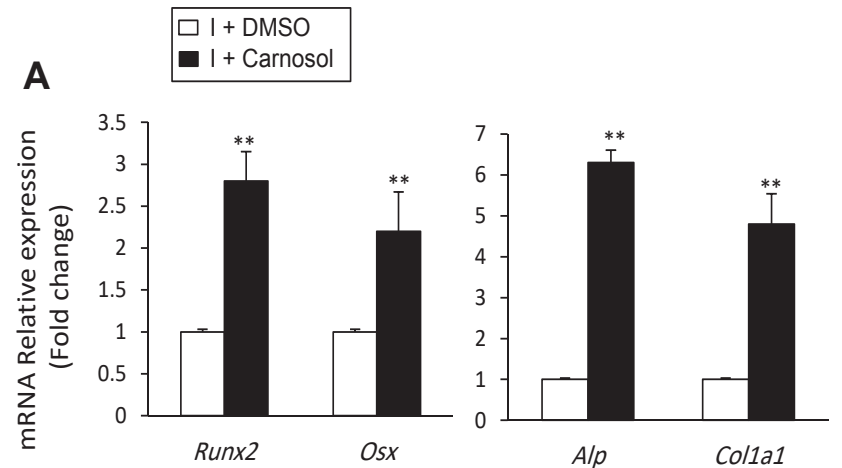

B

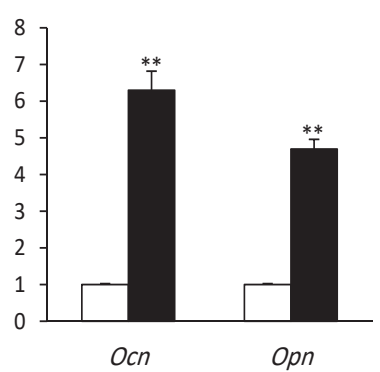

Fig. 2. Carnosol induces the expression of osteoblast-related genes. (A) Carnosol upregulated the gene expression of early (Runx2, Osx, Alp, and Col1a1) and late osteogenic factors (Ocn and Opn) in mouse bone marrowderived mesenchymal stem cells (mBM$\mathrm{SCs}$ ) during osteogenesis. mBMSCs were induced to osteoblast differentiation using OIM in the absence (I+DMSO) or the presence of $10 \mu \mathrm{M}$ carnosol (I+Carnosol) for 12 days. Gene expression values were normalization to reference genes and represented as fold change over induced cells without carnosol. (B) Analysis of carnosol-induced osteoblast-related genes in mBMSCs during osteogenesis using qPCR-based osteogenic gene array assay. The pie chart showed the percentage of upregulated genes as categorized by their osteogenic functions according to the data in Table 1. Values are mean \pm standard deviation of three independent experiments $\left({ }^{* *} \mathrm{p}<0.005\right.$ compared to I+DMSO). OIM, Osteogenic-induction medium. 
upregulated the gene expression of Runx2, Alp and Ocn during the time course of osteoblast differentiation of mBMSCs (Supplementary Fig. 1B). QPCR-based osteogenic gene array analysis of carnosol-induced osteogenesis in mBMSCs revealed significant up-regulation of the expression of genes related to osteoblast differentiation and ossification and matrix formation by $31.8 \%$ and $27.3 \%$ respectively (Fig. 2 B, Table 1 ).

\section{Carnosol inhibits the differentiation of mBMSCs into adipocytes}

To examine, whether carnosol has the potential to direct the differentiation of mBMSCs into osteoblast versus adipocyte, we study the effect of carnosol on adipogenesis of mBMSCs. Carnosol showed to exert dose-dependent inhibitory effect on the differentiation of mBMSCs into adipocyte as assessed by for lipid accumulation and its quantification (Fig. 3A, B). In addition, Carnosol inhibited the capacity of mBMSCs to differentiate into adipocyte at different time points during the whole course of adi- pogenesis as assessed by quantitative Oil Red O staining (Supplementary Fig. 2A). At the molecular level, carnosol significantly down-regulated the mRNA expression of both early (Ppary2 and $C / e b p \alpha$ ) and late ( $(P 2$ and $L p I$ ) adipogenic genes in mBMSCs as assessed by qPCR analysis (Fig. 3C). Furthermore, carnosol down-regulated both Ppar 2 and aP2 at different time points during the course of adipogenesis in mBMSCs (Supplementary Fig. 2B).

\section{Carnosol stimulates BMP2-induced osteogenesis in mBMSCs}

To understand the mechanism underlying the stimulatory effect of carnosol on osteogenesis, we studied the effect of carnosol on signaling molecules known to induce osteogenesis and bone formation (including TGF $\beta 1$, BMP2, IGF-1, PDGF, and bFGF). Interestingly, carnosol significantly stimulated BMP2-induced osteogenesis compared to other factors, as assessed by ALP activity measurements (Fig. 4A). This finding was also confirmed for

Table 1. Upregulated osteogenic genes by carnosol during osteogenic differentiation of mBMSCs

\begin{tabular}{|c|c|c|}
\hline Gene name & Gene symbol & Fold change \\
\hline \multicolumn{3}{|l|}{ Ossification and matrix molecules } \\
\hline Alkaline phosphatase, liver/bone/kidney & Alpl & 10.5 \\
\hline Bone gamma carboxyglutamate protein & Bglap & 8.3 \\
\hline Biglycan & Bgn & 3.8 \\
\hline Collagen type I alpha 1 & Col1a1 & 5.7 \\
\hline Collagen type $V$ alpha 1 & Col5a1 & 4.8 \\
\hline Secreted phosphoprotein 1 (Osteopontin) & Spp1 & 3.2 \\
\hline \multicolumn{3}{|l|}{ Osteoblast differentiation } \\
\hline Bone morphogenetic protein 2 & Bmp2 & 5.2 \\
\hline Bone morphohenitic protein 4 & Bmp4 & 6.4 \\
\hline Bone morphogenetic protein receptor, type $1 \mathrm{~A}$ & Bmpr1a & 2.1 \\
\hline Bone morphohenitic protein receptor, type 1B & Bmpr1a & 6.3 \\
\hline Distal-less homeobox 5 & Dlx5 & 7.3 \\
\hline Runt related transcription factor 2 & Runx2 & 4.6 \\
\hline Sp7 transcription factor 7 & Sp7 & 2.3 \\
\hline \multicolumn{3}{|l|}{ Cell adhesion molecules } \\
\hline Fibronectin 1 & Fn1 & 3.4 \\
\hline Integrin beta 1 (fibronectin receptor beta) & $\operatorname{ltg} b 1$ & 2.3 \\
\hline Integrin alpha 2 & Itga2 & 2.8 \\
\hline Integrin alpha $2 \mathrm{~b}$ & $\operatorname{ltga} 2 b$ & 3.4 \\
\hline \multicolumn{3}{|l|}{ Osteogenic growth factors } \\
\hline Fibroblast growth factor receptor 2 & Fgfr2 & 3.9 \\
\hline Insulin-like growth factor 1 & $\operatorname{lgf1}$ & 8.4 \\
\hline Insulin-like growth factor I receptor & $\operatorname{lgf1r}$ & 4.3 \\
\hline Platelet derived growth factor alpha & Pdgfa & 5.2 \\
\hline Vascular endothelial growth factor A & Vegfa & 3.1 \\
\hline
\end{tabular}

Cells were induced to differentiate into osteoblast in the absence (control) or the presence of carnosol (10 $\mu \mathrm{M})$. After 6 days of induction, total RNA were extracted and Mouse osteogenesis RT2 Profiler PCR array was performed was performed using quantitative PCR method. Up-regulated genes by carnosol were normalized to reference genes and represented as fold change over control cells without carnosol. Values are mean of 3 independent experiments. mBMSCs, mouse bone marrow-derived mesenchymal stem cells. 
A
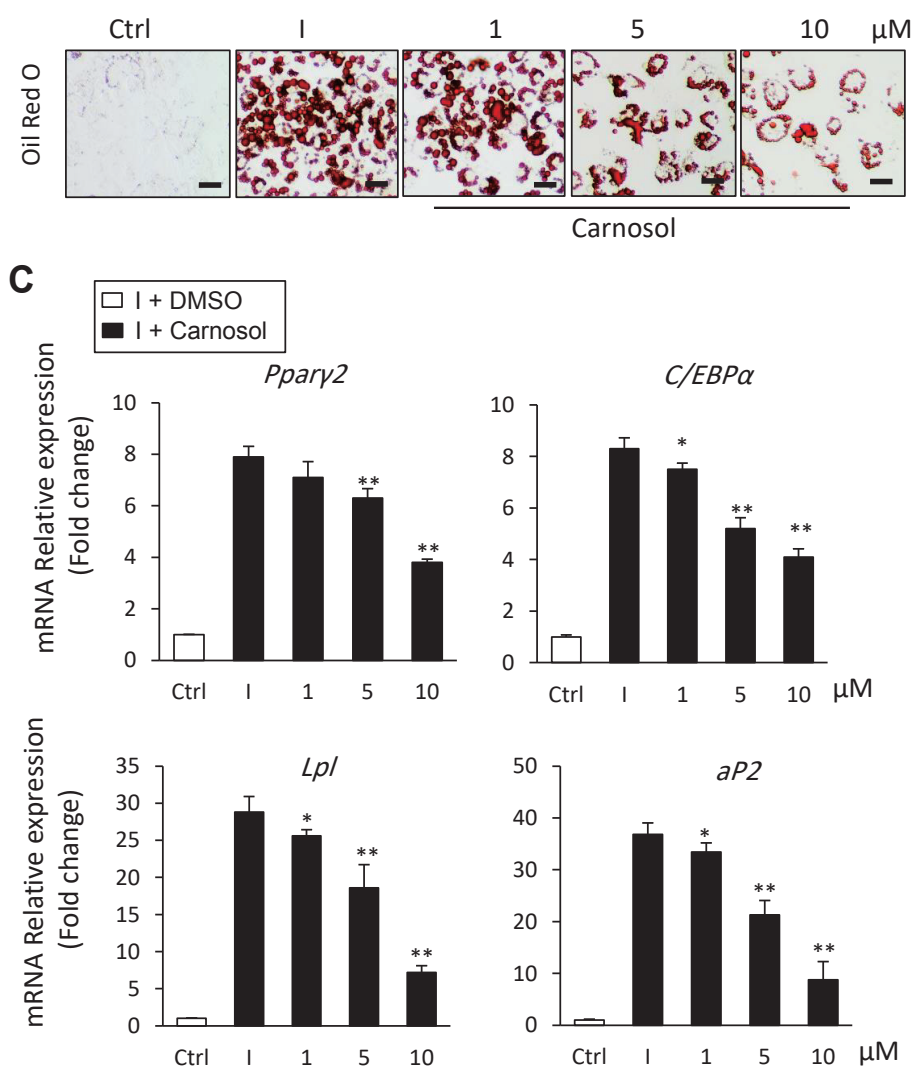

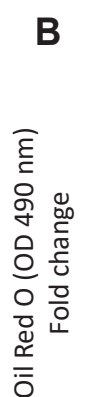

$C / E B P \alpha$
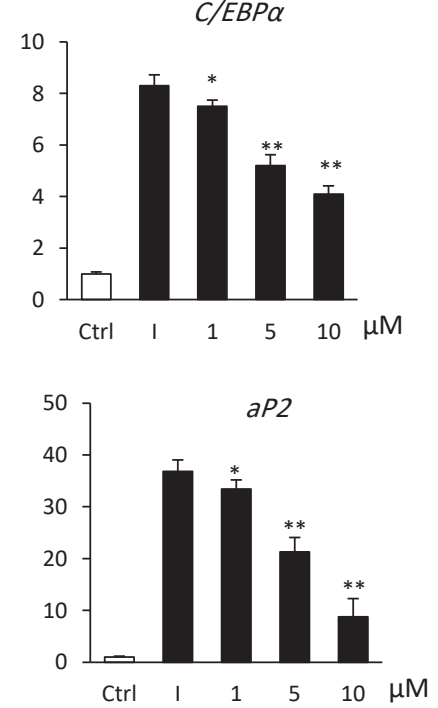

Fig. 3. Carnosol inhibits the differentiation of mouse bone marrow-derived mesenchymal stem cells (mBMSCs) into adipocytes. (A) Inhibitory effect of carnosol on adipogenesis of mBMSCs as measured by Oil Red O staining for fat droplets formation. mBMSCs were induced to differentiated into adipocytes either in the presence of DMSO (I) as control or different concentrations of carnosol. Scale bars $=100 \mu \mathrm{m}$. (B) Quantification of Oil Red O staining after 12 days of differentiation. (C) Quantitative real-time PCR analysis of the adipogenic specific genes expression in the differentiated mBMSCs into adipocytes in the presence of different concentrations of carnosol. Gene expression values were normalized to reference genes and represented as fold inhibition to induced cells without carnosol. Values are mean \pm standard deviation of three independent experiments $\left({ }^{*} \mathrm{p}<0.05,{ }^{* *} \mathrm{p}\right.$ $<0.005$, compared to [l]).

other BMP members (BMP4 and 7) as assessed by significant increased matrix mineralization in treated mBMSCs with BMPs in the presence on carnosol versus cells treated without carnosol (Fig. $4 \mathrm{~B})$. In addition, treatment of mBMSCs with carnosol showed to significantly upregulate the BMP2-induced osteoblastic target genes (Alp, Ocn, Colla1, Runx2, Dlx5, and Msx2) as compared to non-treated cells (Fig. 4C).

\section{The mechanism of carnosol-induced osteogenesis is mediated by BMP signaling pathway}

To investigate, whether the stimulatory effect of carnosol on osteogenesis is mediated via BMP signaling, we first studied the possible activation of BMP2 signaling in mBMSCs by carnosol. Carnosol displayed does-dependent activation of Smad 1/5/8 phosphorylation as assessed by Western blot analysis (Fig. 5A). Furthermore, inhibition of BMP signaling in mBMSCs with a specific BMP1R inhibitor, LDN-193189, showed to significantly inhibit the stimulatory effect of carnosol on BMP-induced Smad $1 / 5 / 8$ phosphorylation as assessed by western blot analysis (Fig. 5B). Consequently, treatment of mBMSCs with LDN-193189 in the presence of carnosol, showed to attenuate the stimulatory effect of carnosol on osteogenesis by $62 \%$ compared to treated cells without inhibitor as measured by the quantitative ALP activity assay (Fig. 5C).

\section{DISCUSSION}

Carnosic acid and its major oxidative derivatives, carnosol are major antioxidant components (> 90\%) of rosemary (Rosmarinus officinalis) that showed many applications in field of nutrition, and health $[24,25]$.

In this study, we identified the medicinally important phytochemical, carnosol as a novel osteogenic factor that promotes the differentiation of BMSCs into osteoblasts, while inhibits their 

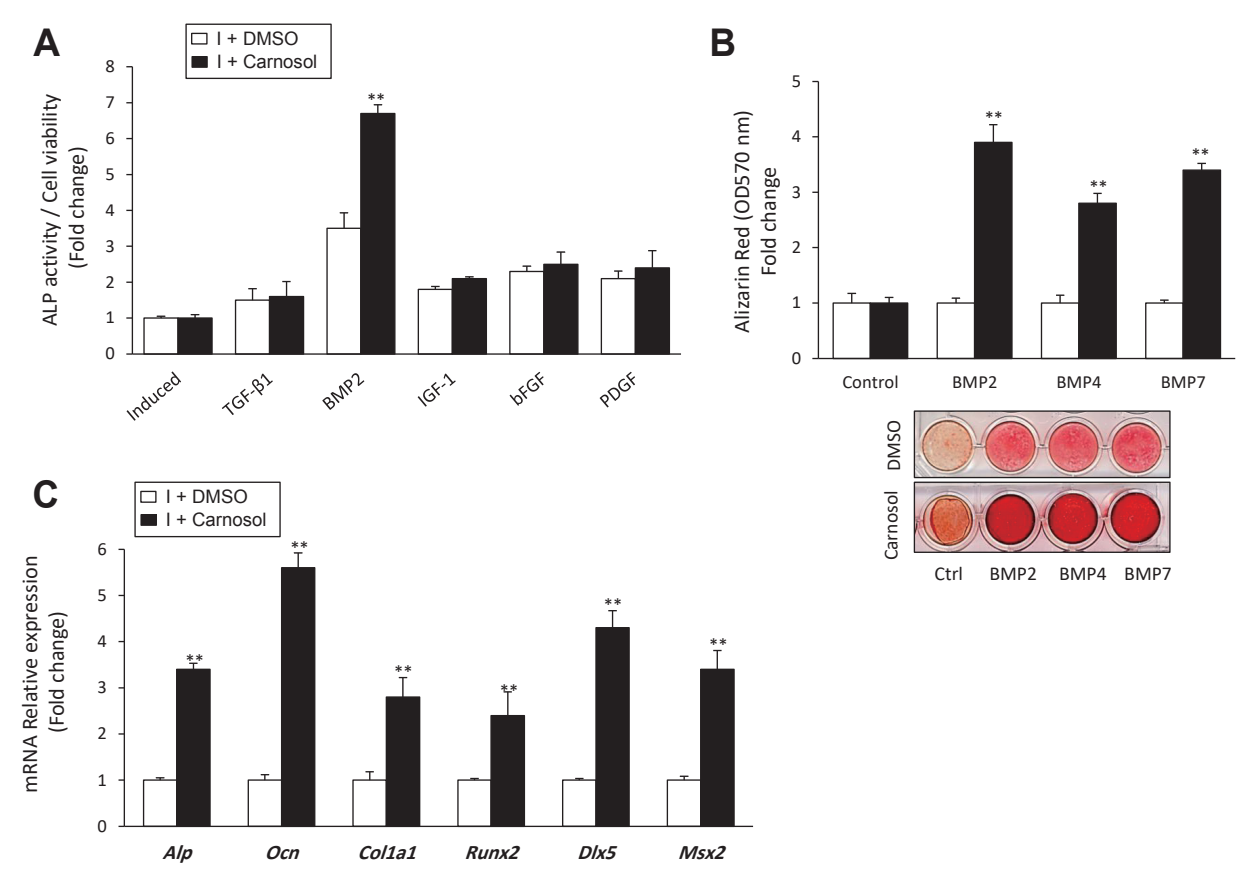

Fig. 4. Carnosol promotes bone morphogenetic protein (BMP)-induced osteogenesis in mouse bone marrow-derived mesenchymal stem cells (mBMSCs). (A) Studying the effect of carnosol on promoting the osteogenic induction of different osteogenic growth factors in mBMSCs. Cells were induced for osteogenesis without (I+DMSO) or with TGF $\beta 1$ (10 ng/ml), BMP2 (100 ng/ml), IGF1 (100 ng/ml), bFGF (100 ng/ml) or PDGF (100 ng/ $\mathrm{ml}$ ) for 6 days in the absence or the presence of $10 \mu \mathrm{M}$ carnosol for 6 days. (B) Effect of carnosol on BMP2, 4, and 7-induced osteogenesis in mBMSCs as measured by quantitative Alizarin Red staining for matrix mineralization after 12 days of induction. Representative images of Alizarin Red staining were shown. (C) Effect of carnosol on inducing the expression of BMP2-upregulated osteogenic gene expression. Gene expression values were normalization to reference genes and represented as fold change over induced cells without carnosol. Values are mean \pm standard deviation of three independent experiments $\left({ }^{* *} \mathrm{p}<0.005\right.$, compared to induced cells without carnosol [I+DMSO]).

differentiation into adipocytes. Our data revealed that the stimulatory effect of carnosol on osteogenesis is mediated via BMPsignaling pathway.

Our data demonstrated that carnosol promoted osteogenic differentiation at the early and late stages by increasing ALP activity and matrix mineralization respectively. In consistent, at the molecular level, carnosol upregulated the expression of early and late osteogenic markers, suggesting the involvement of carnosol in directing the early commitment of BMSCs into osteoblastic cell lineage and promoting mature osteoblast formation.

BMP signaling is an essential pathway that involved in osteoblast differentiation and bone formation. Binding of the osteogenic inducers, BMPs to its serine-threonine receptors (BMP type I receptor and type II receptors) leads to activate the phosphorylation of Smad1/5/8 (phosphorylated R-Smad). Subsequently, phosphorylated R-Smad binds to SMAD4 and co-translocates into the nucleus to activate the expression of main osteogenic transcription factor Runx 2 and osteogenic target genes, i.e., Ocn, Dlx 5 , and Msx2 [26-28]. Our data, identified carnosol as a novel inducer for BMP-induced osteogenesis, via canonical-Smad-dependent pathway in a dose-dependent manner. In addition, the stimulatory effect of carnosol on osteogenesis in mBMSCs was abolished upon the inhibition of BMP signaling by specific BMP1R inhibitor. In this context, the biological effects of carnosol were found to be mediated by several signaling molecules. Carnosol was reported to inhibit cartilage breakdown in osteoarthritic chondrocytes via the inhibition of pro-inflammatory factors [18] and to suppress osteoclast formation and activity by inhibiting NF$\kappa \mathrm{B}$ ligand (RANKL)-induced osteoclast [21]. Carnosol exerts long term antioxidant effect by activating phosphatidylinositol 3-kinase (PI3K)/Akt pathway and HO-1 pathways [29]. Furthermore, many signaling molecules are implicated in the anticancer properties of carnosol including molecules associated with cell cycles including PI3K/Akt, mammalian target of rapamycin (mTOR), and 5-AMP-activated protein kinase (AMPK) and androgen and estrogen receptors $[25,30,31]$.

Our data demonstrated for the first time that carnosol promotes osteogenesis by activating BMP-signaling and upregulating its downstream osteogenic target genes.

However, the mechanism of how carnosol can activate BMP signaling is still unknown. Further studies are required to investigate, whether carnosol can bind to BMP receptors or acting downstream intracellularly to activate $\mathrm{Smad} 4$ protein. In regards to the affinity of carnosol to some cell receptors, carnosol showed to inhibit both prostate and breast cancers by binding and antagonizing estrogen and androgen receptors [32-34].

Our data demonstrated the inhibitory effect of carnosol on adipogenesis by suppressing both early and late stages of adipo- 

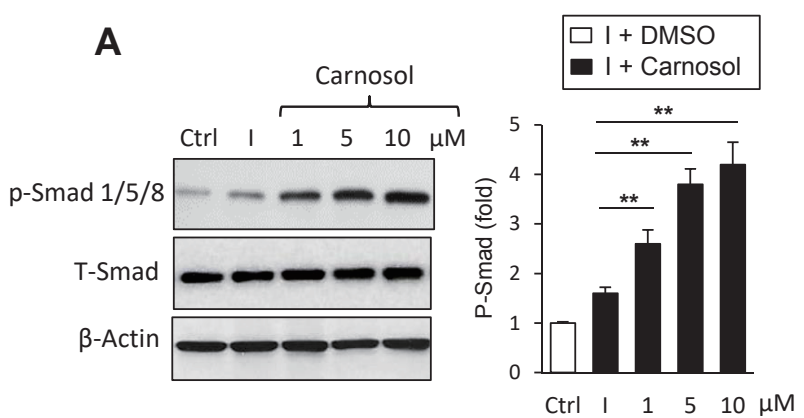

C

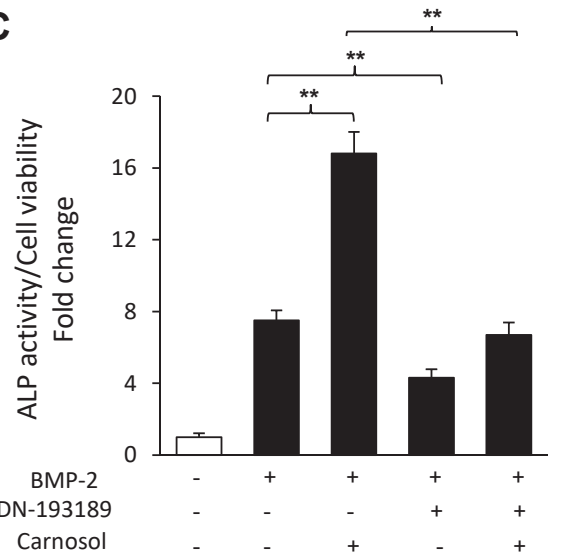

B

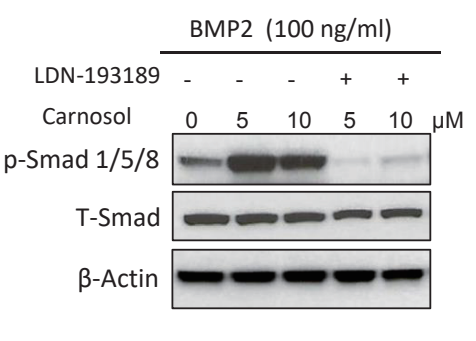

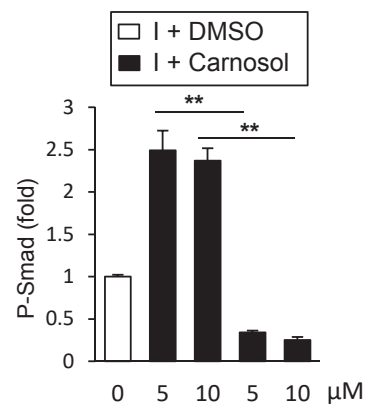

D

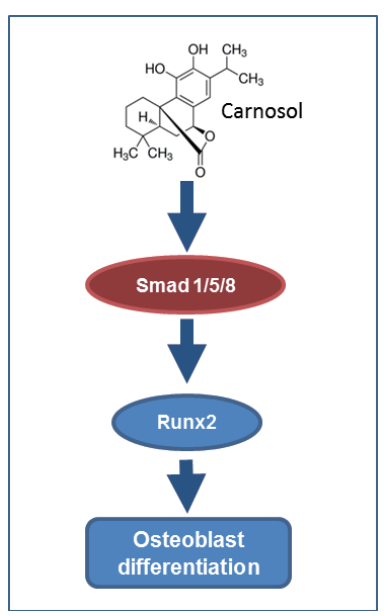

Fig. 5. Carnosol promotes osteoblast differentiation of mouse bone marrow-derived mesenchymal stem cells (mBMSCs) through the activation of bone morphogenetic protein (BMP) signaling pathway. (A) Western blot analysis showing the dose-dependent stimulatory effect of carnosol $(10 \mu \mathrm{M})$ on the activation of Smad1/5/8 phosphorylation in mBMSCs after $10 \mathrm{~min}$. (B) Western blot analysis demonstrating the ability of BMP1R inhibitor (LDN-193189, $10 \mu \mathrm{M}$ ) to inhibit the stimulatory effect of carnosol on BMP-induced Smad1/5/8 phosphorylation in mBMSCs after 10 min of treatment. (C) BMP1R inhibitor (LDN-193189, $10 \mu \mathrm{M}$ ) suppressed the stimulatory effect of carnosol (10 $\mu \mathrm{M})$ on BMP2-induced osteogenesis in mBMSCs as measured by quantitative ALP activity. Cells were pre-treated with LDN-193189, $10 \mu \mathrm{M}$ and induced with BMP2 in the absence or the presence of carnosol $(10 \mu \mathrm{M})$ for 6 days. (D) Schematic diagram illustrating the mechanism of the stimulatory effect of carnosol to on osteogenesis via activating canonical BMP signalling. Values are mean \pm standard deviation of three independent experiments $(* * p<0.005)$. ALP, alkaline phosphatase.

cyte differentiation of BMSCs. In consistent, carnosol inhibits the differentiation of mouse pre-adipocyte 3T3-L1 cells into mature adipocyte by stimulating glutathione metabolism [35]. The antiadipogenic effect of carnosol was revealed by its capacity to protect lipids from oxidation in vitro [24], and to reduce lipid absorption in mice by inhibiting pancreatic lipase(s) [36]. In addition, administration of carnosol to diabetic rats showed to excrete antidiabetic effects by reducing blood glucose, triglyceride and total cholesterol in association with reduced plasma levels of oxidative stress [37].

The lineage specific differentiation of BMSCs into osteoblasts or adipocytes is controlled by several signaling molecules including Wnt, Notch, BMPs and TGF- $\beta 1[3,8,38,39]$. In this context, we recently reported that the adipocytes in bone marrow microenvironment exerts paracrine inhibitory effects on osteogenesis via suppressing BMP-induced osteogenesis [40]. Thus, the inhibitory effect of carnosol on adipogenesis of BMSCs is beneficiary for the stimulatory effect of carnosol on BMP-signaling activation and subsequently promoting osteoblast differentiation.

Phytochemicals are renewable source of compounds with therapeutic potential in many metabolic diseases. In this study, we identified carnosol as a novel osteogenic phytochemical with a high potential to direct the differentiation of BMSCs into osteoblastic cell lineage versus adipocytic cell lineage. The stimulatory effect of carnosol on osteogenesis showed to be mediated via BMP signaling-dependent mechanism (Fig. 5D). Thus, our data provide carnosol as a novel renewable source of phytochemical that plausibly can be used directly as an osteoanabolic drug or as raw material to develop drug for enhancing bone formation in many bone-loss related diseases.

\section{ACKNOWLEDGEMENTS}

The Authors acknowledge the Deanship of Scientific Research at King Faisal University, Saudi Arabia for the financial support 
under Nasher Track (Grant No. 186301).

\section{CONFLICTS OF INTEREST}

The author declares no conflicts of interest.

\section{SUPPLEMENTARY MATERIALS}

Supplementary data including one table and two figures can be found with this article online at https://doi.org/10.4196/ kjpp.2021.25.3.197.

\section{REFERENCES}

1. Kim JM, Lin C, Stavre Z, Greenblatt MB, Shim JH. Osteoblast-osteoclast communication and bone homeostasis. Cells. 2020;9:2073.

2. Russow G, Jahn D, Appelt J, Märdian S, Tsitsilonis S, Keller J. Anabolic therapies in osteoporosis and bone regeneration. Int J Mol Sci. 2018;20:83.

3. Abdallah BM, Jafari A, Zaher W, Qiu W, Kassem M. Skeletal (stromal) stem cells: an update on intracellular signaling pathways controlling osteoblast differentiation. Bone. 2015;70:28-36.

4. Abdallah BM, Kassem M. Human mesenchymal stem cells: from basic biology to clinical applications. Gene Ther. 2008;15:109-116.

5. Park-Min KH. Metabolic reprogramming in osteoclasts. Semin Immunopathol. 2019;41:565-572.

6. Abdallah BM, Kassem M. The use of mesenchymal (skeletal) stem cells for treatment of degenerative diseases: current status and future perspectives. J Cell Physiol. 2009;218:9-12.

7. Bianco P, Robey PG. Skeletal stem cells. Development. 2015;142: 1023-1027.

8. Abdallah BM, Kassem M. New factors controlling the balance between osteoblastogenesis and adipogenesis. Bone. 2012;50:540-545.

9. Bartold M, Gronthos S, Haynes D, Ivanovski S. Mesenchymal stem cells and biologic factors leading to bone formation. J Clin Periodontol. 2019;46 Suppl 21:12-32.

10. Ditano-Vázquez P, Torres-Peña JD, Galeano-Valle F, Pérez-Caballero AI, Demelo-Rodríguez P, Lopez-Miranda J, Katsiki N, DelgadoLista J, Alvarez-Sala-Walther LA. The fluid aspect of the mediterranean diet in the prevention and management of cardiovascular disease and diabetes: the role of polyphenol content in moderate consumption of wine and olive oil. Nutrients. 2019;11:2833.

11. Gotsis E, Anagnostis P, Mariolis A, Vlachou A, Katsiki N, Karagiannis A. Health benefits of the Mediterranean Diet: an update of research over the last 5 years. Angiology. 2015;66:304-318.

12. Chang CH, Chyau CC, Hsieh CL, Wu YY, Ker YB, Tsen HY, Peng RY. Relevance of phenolic diterpene constituents to antioxidant activity of supercritical $\mathrm{CO}_{2}$ extract from the leaves of rosemary. Nat Prod Res. 2008;22:76-90.

13. Andrade JM, Faustino C, Garcia C, Ladeiras D, Reis CP, Rijo P. Rosmarinus officinalis L.: an update review of its phytochemistry and biological activity. Future Sci OA. 2018;4:FSO283.
14. Weckesser S, Engel K, Simon-Haarhaus B, Wittmer A, Pelz K, Schempp CM. Screening of plant extracts for antimicrobial activity against bacteria and yeasts with dermatological relevance. Phytomedicine. 2007;14:508-516.

15. Samarghandian S, Azimi-Nezhad M, Farkhondeh T. Anti-carcinogenic effects of carnosol- an updated review. Curr Drug Discov Technol. 2018;15:32-40.

16. González-Vallinas M, Reglero G, Ramírez de Molina A. Rosemary (Rosmarinus officinalis L.) extract as a potential complementary agent in anticancer therapy. Nutr Cancer. 2015;67:1221-1229.

17. Lin C, Zhang X, Su Z, Xiao J, Lv M, Cao Y, Chen Y. Carnosol improved lifespan and healthspan by promoting antioxidant capacity in Caenorhabditis elegans. Oxid Med Cell Longev. 2019;2019:5958043.

18. Sanchez C, Horcajada MN, Membrez Scalfo F, Ameye L, Offord E, Henrotin Y. Carnosol inhibits pro-inflammatory and catabolic mediators of cartilage breakdown in human osteoarthritic chondrocytes and mediates cross-talk between subchondral bone osteoblasts and chondrocytes. PLoS One. 2015;10:e0136118.

19. Schwager J, Richard N, Fowler A, Seifert N, Raederstorff D. Carnosol and related substances modulate chemokine and cytokine production in macrophages and chondrocytes. Molecules. 2016;21:465.

20. Oliviero F, Scanu A, Zamudio-Cuevas Y, Punzi L, Spinella P. Antiinflammatory effects of polyphenols in arthritis. J Sci Food Agric. 2018;98:1653-1659.

21. Li Y, Lin S, Liu P, Huang J, Qiu J, Wen Z, Yuan J, Qiu H, Liu Y, Liu Q, Zhou T, Luo P, Guo H, Ma Y, Guo D, Mo G, Tang Y, Xu L, Liang D, $\mathrm{Xu}$ J, et al. Carnosol suppresses RANKL-induced osteoclastogenesis and attenuates titanium particles-induced osteolysis. J Cell Physiol. 2021;236:1950-1966.

22. Abdallah BM, Alzahrani AM, Abdel-Moneim AM, Ditzel N, Kassem M. A simple and reliable protocol for long-term culture of murine bone marrow stromal (mesenchymal) stem cells that retained their in vitro and in vivo stemness in long-term culture. Biol Proced Online. 2019;21:3.

23. Abdallah BM, Alzahrani AM, Kassem M. Secreted Clusterin protein inhibits osteoblast differentiation of bone marrow mesenchymal stem cells by suppressing ERK1/2 signaling pathway. Bone. 2018;110:221-229.

24. Loussouarn M, Krieger-Liszkay A, Svilar L, Bily A, Birtić S, Havaux M. Carnosic acid and carnosol, two major antioxidants of rosemary, act through different mechanisms. Plant Physiol. 2017;175:13811394.

25. Johnson JJ. Carnosol: a promising anti-cancer and anti-inflammatory agent. Cancer Lett. 2011;305:1-7.

26. Wu M, Chen G, Li YP. TGF- $\beta$ and BMP signaling in osteoblast, skeletal development, and bone formation, homeostasis and disease. Bone Res. 2016;4:16009.

27. Franceschi RT, Xiao G. Regulation of the osteoblast-specific transcription factor, Runx2: responsiveness to multiple signal transduction pathways. J Cell Biochem. 2003;88:446-454.

28. Halloran D, Durbano HW, Nohe A. Bone morphogenetic protein-2 in development and bone homeostasis. J Dev Biol. 2020;8:19.

29. Martin D, Rojo AI, Salinas M, Diaz R, Gallardo G, Alam J, De Galarreta CM, Cuadrado A. Regulation of heme oxygenase-1 expression through the phosphatidylinositol 3-kinase/Akt pathway and the Nrf2 transcription factor in response to the antioxidant 
phytochemical carnosol. J Biol Chem. 2004;279:8919-8929.

30. Allegra A, Tonacci A, Pioggia G, Musolino C, Gangemi S. Anticancer activity of Rosmarinus officinalis L.: mechanisms of action and therapeutic potentials. Nutrients. 2020;12:1739.

31. Chun KS, Kundu J, Chae IG, Kundu JK. Carnosol: a phenolic diterpene with cancer chemopreventive potential. J Cancer Prev. 2014;19:103-110.

32. Vergara D, Simeone P, Bettini S, Tinelli A, Valli L, Storelli C, Leo S, Santino A, Maffia M. Antitumor activity of the dietary diterpene carnosol against a panel of human cancer cell lines. Food Funct. 2014;5:1261-1269.

33. Johnson JJ, Syed DN, Heren CR, Suh Y, Adhami VM, Mukhtar H. Carnosol, a dietary diterpene, displays growth inhibitory effects in human prostate cancer $\mathrm{PC}_{3}$ cells leading to $\mathrm{G}_{2}$-phase cell cycle arrest and targets the 5'-AMP-activated protein kinase (AMPK) pathway. Pharm Res. 2008;25:2125-2134.

34. Johnson JJ, Syed DN, Suh Y, Heren CR, Saleem M, Siddiqui IA, Mukhtar H. Disruption of androgen and estrogen receptor activity in prostate cancer by a novel dietary diterpene carnosol: implications for chemoprevention. Cancer Prev Res (Phila). 2010;3:11121123.

35. Takahashi T, Tabuchi T, Tamaki Y, Kosaka K, Takikawa Y, Satoh
T. Carnosic acid and carnosol inhibit adipocyte differentiation in mouse 3T3-L1 cells through induction of phase2 enzymes and activation of glutathione metabolism. Biochem Biophys Res Commun. 2009;382:549-554.

36. Ninomiya K, Matsuda H, Shimoda H, Nishida N, Kasajima N, Yoshino T, Morikawa T, Yoshikawa M. Carnosic acid, a new class of lipid absorption inhibitor from sage. Bioorg Med Chem Lett. 2004;14:1943-1946.

37. Samarghandian S, Borji A, Farkhondeh T. Evaluation of antidiabetic activity of carnosol (phenolic diterpene in rosemary) in streptozotocin-induced diabetic rats. Cardiovasc Hematol Disord Drug Targets. 2017;17:11-17.

38. Abdallah BM, Ali EM. 5'-hydroxy Auraptene stimulates osteoblast differentiation of bone marrow-derived mesenchymal stem cells via a BMP-dependent mechanism. J Biomed Sci. 2019;26:51.

39. Li Y, Jin D, Xie W, Wen L, Chen W, Xu J, Ding J, Ren D. PPAR- $\gamma$ and Wnt regulate the differentiation of MSCs into adipocytes and osteoblasts respectively. Curr Stem Cell Res Ther. 2018;13:185-192.

40. Abdallah BM. Marrow adipocytes inhibit the differentiation of mesenchymal stem cells into osteoblasts via suppressing BMP-signaling. J Biomed Sci. 2017;24:11. 